\title{
LncRNA39026 Enhances Tomato Resistance to Phytophthora infestans by Decoying miR168a and Inducing PR Gene Expression
}

\author{
Xinxin Hou, ${ }^{1}$ Jun Cui, ${ }^{1}$ Weiwei Liu, ${ }^{1}$ Ning Jiang, ${ }^{1}$ Xiaoxu Zhou, ${ }^{1}$ Hongyan Qi, ${ }^{2}$ Jun Meng,,$+\dagger$ and Yushi Luan ${ }^{1, \dagger}$ \\ ${ }^{1}$ School of Bioengineering, Dalian University of Technology, Dalian 116024, China \\ ${ }^{2}$ College of Horticulture, Shenyang Agricultural University/Key Laboratory of Protected Horticulture, Ministry of Education/Northern National \\ \& Local Joint Engineering Research Center of Horticultural Facilities Design and Application Technology (Liaoning), Shenyang \\ 110866, China \\ ${ }^{3}$ School of Computer Science and Technology, Dalian University of Technology, Dalian 116024, China \\ Accepted for publication 19 December 2019.
}

\begin{abstract}
Our previous study has indicated that a long noncoding RNA (lncRNA), lncRNA39026, can be responsive to Phytophthora infestans infection. However, the function and regulation mechanism of lncRNA39026 during tomato resistance to P. infestans are unknown. In this study, an IncRNA39026 sequence was cloned from tomato Zaofen No. 2, and this sequence contained an endogenous target mimicry for miR168a, which might suppress the expression of miR168a. LncRNA39026 was strongly downregulated at $3 \mathrm{~h}$ in the tomato plants infected with $P$. infestans, and its expression level displayed a negative correlation with the expression level of miR168a and a positive

$P$. infestans, decreased level of miR168a, and increased level of SlAGO1, whereas the resistance was impaired, level of miR168a was increased, and level of SlAGO1 was decreased after lncRNA39026 silencing. In addition, IncRNA39026 could also induce the expression of pathogenesis-related (PR) genes, as shown by increased and decreased expression levels of PR genes in tomato plants with overexpressed and silenced lncRNA39026, respectively. The result demonstrated that lncRNA39026 might function to decoy miR168a and affect the expression of PR genes in tomato plants, increasing resistance to disease.
\end{abstract} correlation with the expression levels of SlAGO1 genes (target gene of miR168a) upon $P$. infestans infection. Tomato plants in which lncRNA39026 was overexpressed displayed enhanced resistance to
Keywords: IncRNA, miRNA, pathogenesis-related protein, Phytophthora infestans, tomato
Tomato, which is cultivated worldwide, is an important economic crop, and its yield has achieved 182 million ton, worth $>\$ 60$ billion in 2017 (Baysal-Gurel et al. 2015; Gao et al. 2019). However, tomato plants cultivated in the field are at risk from various pathogens during growth and development. Late blight, caused by an oomycete pathogen Phytophthora infestans in high-humidity and low-temperature conditions, is thought to be one of the most devastating diseases in tomato and leads to huge economic losses in tomato production (Ren et al. 2019; Zhang et al. 2013a). Therefore, to prevent damage and reduce losses caused by late blight, it is necessary to study defense mechanisms in tomato resistance to P. infestans.

Noncoding RNAs (ncRNAs), which have little or no ability to code protein, are involved in various biological processes by serving as regulatory molecules, including long noncoding RNAs (lncRNAs) and microRNAs (miRNAs) (Wang et al. 2016). LncRNAs, which are $>200$ nt long, function by interacting with miRNAs, affecting gene expression (Franco-Zorrilla et al. 2007; Wierzbicki 2012). It has been demonstrated that lncRNAs play indispensable roles in many plant biology processes, including resistance to pathogen infection (Zhang et al. 2016). For example,

†Corresponding authors: Y. Luan; ysluan@dlut.edu.cn, and J. Meng; mengjun@dlut.edu.cn

Funding: This work is supported by the National Natural Science Foundation of China grant numbers 31872116 and 61872055

*The $\boldsymbol{e}$-Xtra logo stands for "electronic extra" and indicates that two supplementary figures and one supplementary table are published online.

The author(s) declare no conflict of interest.

C 2020 The American Phytopathological Society
lncRNAs have an effect on Arabidopsis thaliana against Fusarium oxysporum infection (Zhu et al. 2014), wheat against powdery mildew fungi infection (Zhang et al. 2013b), cotton against Verticillium dahliae infection (Zhang et al. 2018), and others. In tomato, many lncRNAs have been found to be involved in host response to potato spindle tuber viroid (Zheng et al. 2017). In the tomato-yellow leaf curl virus (TYLCV) interaction, tomato lncRNAs slylnc0195 and slylnc1077 may regulate miRNA expression to affect tomato resistance (Wang et al. 2015). Another lncRNA, S-slylnc0957, is silenced in susceptible tomato plants by virusinduced gene silencing (VIGS), resulting in increased resistance to TYLCV (Wang et al. 2018). In addition, TYLCV intergenic small interfering RNAs (siRNAs) are transferred into tomato plants to target a host lncRNA to modulate disease symptoms (Yang et al. 2019a). MiRNAs are 20- to 25-nt ncRNAs and emerge as indispensable regulators in plant resistance response by regulating the expression of endogenous genes at the transcriptional and posttranscriptional levels (Li et al. 2014; Voinnet 2009). For example, miR168 is a class of conserved miRNAs in plants and participates in host defense by regulating its target gene AGOl (Pertermann et al. 2018; Vaucheret 2009). A. thaliana miR168 is responsive to many pathogen treatments, such as Turnip crinkle virus, Cucumber mosaic virus, Crucifer-infecting Tobamovirus (Várallyay and Havelda 2013), and F. oxysporum elicitors (Baldrich et al. 2014). In tomato, the miR168 family contains two members, miR168a and miR168b. Among them, only miR168a was responsive to $P$. infestans infection by targeting SlAGO1 (Solyc06g072300.2.1) gene (Cui et al. 2019a; Luan et al. 2015).

Accumulated evidence has demonstrated that lncRNAs emerged as a new regulator of miRNA activity by acting as endogenous target mimics (eTMs) for miRNAs (Wu et al. 2013; Wang et al. 2019). For example, in maize, lncRNA PILNCR1 regulates $\mathrm{PHO} 2$ expression by decoying miR399 to modulate Pi-deficiency stress (Du et al. 
2018). Moreover, cotton lncRNAs lnc_973 and lnc_253 respond to salt stress by acting as eTMs of miR399 and miR156e (Deng et al. 2018). Wang et al. (2017) found that rice lncRNA eTM160 can serve as a decoy for miR160 to regulate reproductive development. However, the finding that lncRNAs participate in plant responses to pathogens by acting as eTMs is rarely reported. In tomato infection by TYLCV, slylnc0195 and slylnc1077, which contain eTMs for miR166 and miR399, suppress their expression, respectively, to affect plant defense response (Wang et al. 2015).

Pathogenesis-related (PR) genes act as markers in defense-related signaling pathways and are involved in plant resistance to pathogens (Dai et al. 2016). Some apple PR genes are induced in response to inoculation with the pathogen Erwinia amylovora, the causal agent of fire blight (Hassani et al. 2016). The resistant tomato plants respond quickly to Meloidogyne incognita infection by increasing the accumulation of transcripts of PR genes with the pathogen invasion (Lavrova et al. 2017). In our previous studies, we showed that some WRKY transcription factors promote increased accumulation of PR genes in tomato infected with $P$. infestans (Cui et al. 2018; Hong et al. 2018; Li et al. 2015). Likewise, lncRNAs can also affect the expression of PR genes. A. thaliana lncRNA ELENA1 can induce PRl gene expression to increase plant resistance to Pseudomonas syringe pv. tomato DC3000 (Seo et al. 2017).

In our previous studies, IncRNA33732 was found to induce respiratory burst oxidase ( $\mathrm{RBOH})$ expression, which can increase $\mathrm{H}_{2} \mathrm{O}_{2}$ accumulation to increase tomato defense response to P. infestans (Cui et al. 2019b). Moreover, another tomato lncRNA23468 may decoy miR482b to regulate the expression of $N B S$ - $L R R$ genes, affecting plant resistance to pathogens (Jiang et al. 2019). Also, a resistance-related lncRNA39026 was isolated in tomato plants infected with P. infestans (Cui et al. 2019a). Nevertheless, the particular function and regulation mechanism of lncRNA39026 are unknown. In this study, lncRNA39026 was cloned from tomato plants, its expression level was analyzed in tomato response to $P$. infestans, and its function was explored by gain- and loss-of-function of lncRNAs. Subsequently, it was predicted and demonstrated that lncRNA39026 might act as an eTM for miR168a. Meanwhile, we also measured the expression of PR genes in the tomato plants with overexpressed and silenced lncRNA39026. Our studies will help us elucidate the lncRNAmiRNA interaction and future breeding for molecular-based disease resistance tomato.

\section{MATERIALS AND METHODS}

Plant growth and $\boldsymbol{P}$. infestans inoculation. Solanum lycopersicum Zaofen No. 2, a cultivar tomato bred by the Institute of Vegetables and Flowers, Chinese Academy of Agricultural Sciences, Beijing, China, is a susceptible accession to $P$. infestans. The tomato seeds were germinated with moist filter paper under $25^{\circ} \mathrm{C}$ in dark. Then the sprouted seeds were transferred to soil and grown in a greenhouse with $16 \mathrm{~h}$ light and $8 \mathrm{~h}$ dark, under $25 \pm 3^{\circ} \mathrm{C}$. Tomato seedlings at the four- to five-leaf stage were sprayed with $P$. infestans spore suspension, whose concentration was $1 \times 10^{6}$ zoospores $/ \mathrm{ml}$, then placed in the dark with a $100 \%$ relative humidity environment at $20 \pm 1^{\circ} \mathrm{C}$. The control plants were treated with sterile water and placed under the same conditions. The leaves were collected at $0,3,6,12,24,48$, and $72 \mathrm{~h}$ postinoculation (hpi). All samples were frozen quickly in liquid nitrogen and stored at $-80^{\circ} \mathrm{C}$ until RNA isolation.

RNA isolation and reverse transcription. RNAiso Plus (TaKaRa, Dalian, China) was used to extract the total RNAs from tomato leaves, and TransScript Green Two-Step RT-qPCR SuperMix and TransScript miRNA First-Strand cDNA Synthesis SuperMix (Transgen Biotech, Beijing, China) was performed to reverse transcription according to manufacturer's instructions.

Cloning of IncRNA39026 and construction of the overexpression plasmid. To clone lncRNA39026, a pair of primers
(39026FP and 39026RP) were designed according to the tomato genome information (https://solgenomics.net/) and tomato lncRNA sequences identified in our previous study (Cui et al. 2019a). The complementary DNA from tomato leaves was used as a template in PCR amplification. The PCR product of lncRNA39026 was isolated and cloned into the PCR cloning vector. The positive clones were sequenced. The correctly identified sequence was cloned into pBI121 plasmid and controlled by the strong constitutive CaMV35S promoter. The overexpression plasmid was transformed into Agrobacterium tumefaciens GV3101 by freeze-thaw method, as described by Jiang et al. (2018a).

Identification of eTM in IncRNA39026. To identify whether lncRNA39026 could decoy miRNAs in tomato, we analyzed the eTM sites for miRNAs in lncRNA39026. Tomato miRNAs were obtained from miRbase (http://www.mirbase.org/), and the prediction of the eTM sites was performed according to the rules described by Jiang et al. (2019).

VIGS constructs and Agrobacterium infiltration. To construct VIGS for lncRNA39026, we used tobacco rattle virus (TRV)-based vectors (TRV1 and TRV2) as described by Cui et al. (2019b). The specific primers for VIGS-lncRNA39026 are listed in Supplementary Table S1. TRV2-lncRNA39026 was transformed into A. tumefaciens GV3101. After mixing in the same ratio, A. tumefaciens carrying TRV1 and TRV2 vectors were infiltrated into the leaves of three-leaf-stage tomato seedlings. Then the tomato plants were placed under $20^{\circ} \mathrm{C}$ for 21 days (Cui et al. 2019b). In addition, $A$. tumefaciens harboring overexpression plasmid was infiltrated into $S$. lycopersicum Zaofen No. 2 leaves, and the leaves were collected every day for subsequent experiments.

Disease resistance analysis. For tomato plants with overexpressed or silenced lncRNA39026, the leaves were inoculated with $20 \mu \mathrm{l}$ of a $P$. infestans zoospore suspension $\left(10^{6}\right.$ zoospores $\left./ \mathrm{ml}\right)$ according to the method of Jiang et al. (2018b). The whole plants were sprayed with $P$. infestans spore suspension. After 5 days of treatment, the leaf symptoms were observed, the diameter of the lesions was counted, and the leaves were stained with trypan blue solution according to the method described by $\mathrm{Li}$ et al. (2015). To measure the accumulation of $\mathrm{H}_{2} \mathrm{O}_{2}$ and $\mathrm{O}_{2}^{-}$in treated leaves, diamino benzidine staining and nitro blue tetrazolium staining were performed as described by $\mathrm{Li}$ et al. (2015). In our previous study, the expression levels of superoxide dismutase (SOD, Solyc11g066390.1) and peroxidase (POD, Solyc07g052510.2) increase to enhance reactive oxygen species (ROS) scavenging activity, leading to greater tomato resistance to $P$. infestans (Cui et al. 2018; Li et al. 2015). So, these two genes were chosen to analyze their expression levels. In addition, we analyze the expression levels of eight other members of the tomato SOD gene family in tomato plants with silenced lncRNA39026 (Feng et al. 2016).

Quantitative reverse transcription PCR analysis. To determine the expression levels of the coding genes, lncRNA39026, and its decoyed miRNA, primers were designed with Primer Premier 5.0, and quantitative reverse transcription PCR (RT-qPCR) analysis was performed. Actin was the tomato internal housekeeping gene. Sequences for all the primers are shown in Supplementary Table S1. The data were analyzed by the $2^{-\Delta \Delta \mathrm{Ct}}$ method.

Statistical analysis. All data are expressed as the means \pm SEs of three independent experiments. The Student $t$ test or the Duncan multiple range test was performed in SPSS, and $P<0.05$ was considered statistically significant differences.

\section{RESULTS}

LncRNA39026 is responsive to $P$. infestans infection. The sequence of lncRNA39026 was cloned from tomato Zaofen No. 2, whose length is $614 \mathrm{bp}$. The expression level of lncRNA39026 in $P$. infestans-treated tomato leaves was analyzed by RT-qPCR at 0,3 , $6,12,24,48$, and 72 hpi. Under $P$. infestans induction, the trend of 
the expression of lncRNA39026 first decreased and then increased, reaching the lowest value at 3 hpi and the peak at 24 hpi (Fig. 1). These results indicated that lncRNA39026 was responsive to $P$. infestans infection in tomato plants.

Overexpression of IncRNA39026 increased tomato resistance. To explore the function of lncRNA39026 during tomato resistance to $P$. infestans, overexpressing plasmid pBI121-lncRNA39026 was constructed and transformed into tomato leaves transiently by Agrobacterium-mediated infiltration (Fig. 2A). Introduction of A. tumefaciens harboring pBI121-lncRNA39026 into tomato leaf epidermal cells led to significant upregulation of lncRNA39026 expression, which was steady from 1 to 3 days after agroinfiltration, and at 4 days after agroinfiltration its expression levels decreased. In addition, the tomato leaves might be injured during agroinfiltration, which might affect the resistance to infection (Supplementary Figure S1). To avoid injury, we infected tomato leaves with $P$. infestans at 3 days after agroinfiltration. Compared with tomato plants with overexpressed empty vectors (EVs), the transcript level of lncRNA39026 was upregulated, approximately 56-fold in the tomato plants in which lncRNA39026 was overexpressed at 3 days after agroinfiltration (TOE39026) (Fig. 2B). After 5 days inoculation with $P$. infestans, TOE39026 tomato leaves showed fewer disease symptoms and smaller lesions than EV plants (Fig. 2C and D). These results indicate that overexpression of lncRNA39026 in tomato increased plant resistance to $P$. infestans.

Silencing of IncRNA39026 reduced tomato resistance to $P$. infestans. The VIGS system was constructed to suppress the expression of lncRNA39026, which elucidated its function in tomato-P. infestans interaction. A 370-bp fragment from lncRNA39026 was cloned into a TRV2 vector, and the TRV2 empty vector was performed as the negative control (TRV2). After 21 days of VIGS treatment, RT-qPCR was used to verify decreased efficiency of lncRNA39026 in the tomato plants with silenced lncRNA39026 (VIGS-39026). The results showed that the level of lncRNA39026 was decreased by approximately $80 \%$ compared with TRV2 plants (Fig. 3A). After 5 days of $P$. infestans infection, the disease condition of detached leaves from the TRV2 and VIGS-39026 tomato plants was assessed. The leaves of VIGS-39026 tomato plants displayed more disease symptoms than TRV2 plants (Fig. 3B). Trypan blue staining also revealed that more necrotic cells occurred in the leaves of VIGS-39026 tomato plants (Fig. 3C). Moreover, the VIGS-39026 tomato leaves had longer lesion diameters (Fig. 3D). In the whole-plant infection assay, the silenced plants infected by $P$. infestans developed more disease phenotypes, as shown in Figure 3E and F.

Plants produce ROS, containing $\mathrm{H}_{2} \mathrm{O}_{2}$ and $\mathrm{O}_{2}^{-}$, which are involved in defense mechanism during pathogen infection, but excessive ROS can damage plant cells and decrease resistance (Li et al. 2015). SOD and POD are key regulatory enzymes in the ROS scavenging system, and the expression level of POD (Solyc07g052510.2) was downregulated in VIGS-39026 plants compared with TRV2 plants (Fig. 3G). In nine SOD genes, only Solyc11g066390.1 and Solyc03g062890.2 expressions were inhibited in the tomato plants in which lncRNA39026 is silenced (Fig. $3 \mathrm{H}$ and I), and the expression levels of Solyc01g067740.2, Solyc08g079830.2, Solyc06g048410.2, and Solyc06g049080.2 had no significant changes; only Solyc03g095180.2, Solyc02g021140.2, and Solyc06g048420.1 expressions were upregulated (Supplementary Figure S2). We also detected the accumulation of $\mathrm{H}_{2} \mathrm{O}_{2}$ and $\mathrm{O}_{2}{ }^{-}$in TRV2 and VIGS-39026 plants at 5 dpi through diamino benzidine and nitro blue tetrazolium staining. As shown in Figure $3 \mathrm{~J}$ and $\mathrm{K}$, after infection with $P$. infestans, VIGS-39026 tomato leaves displayed more brown-blue spots and an increase in the accumulation of $\mathrm{H}_{2} \mathrm{O}_{2}$. Meanwhile, after lncRNA39026 silencing in tomato, $\mathrm{O}_{2}^{-}$had obviously accumulated (Fig. 3L and $\mathrm{M}$ ). These results revealed that silencing of lncRNA39026 might inhibit the expression levels of $P O D$ and $S O D$ to affect ROS scavenging activity during tomato resistance to $P$. infestans infection.
LncRNA39026 contained eTM for miR168a. Because of the presence of eTMs for miRNAs on lncRNAs, lncRNAs may decoy miRNAs to suppress their expression and subsequently affect the expression of their target genes. A program was written to predict the eTM in lncRNA39026. The result showed that lncRNA39026 contained an eTM binding site for miR168a from 584 to $604 \mathrm{nt}$. The minimum binding free energy was $-30.4 \mathrm{kcal} /$ mol, and a three-nucleotide bulge located on ninth and 11th positions at the $5^{\prime}$ end of miR168a (Fig. 4A). RT-qPCR results showed that the expression level of miR168a was negatively correlated with the expression level of lncRNA39026. In the tomato leaves infected by $P$. infestans, the trend of the expression of miR168a first increased and then decreased, reaching the peak at 3 and 6 hpi (Figs. 1 and 4B). In the leaves of TOE39026, at 3 days after agroinfiltration, miR168a expression was significantly suppressed, achieved approximately 0.1 -fold, compared with the values of EV leaves (Fig. 4C). Moreover, after 21 days of VIGS treatment, miR168a transcripts increased by approximately $40 \%$ in VIGS-39026 tomato plants compared with TRV tomato plants (Fig. 4D).

Our previous study indicated that miR168a can target SlAGO1 (Solyc06g072300.2.1) at $388 \mathrm{nt}$ in tomato-P. infestans interaction

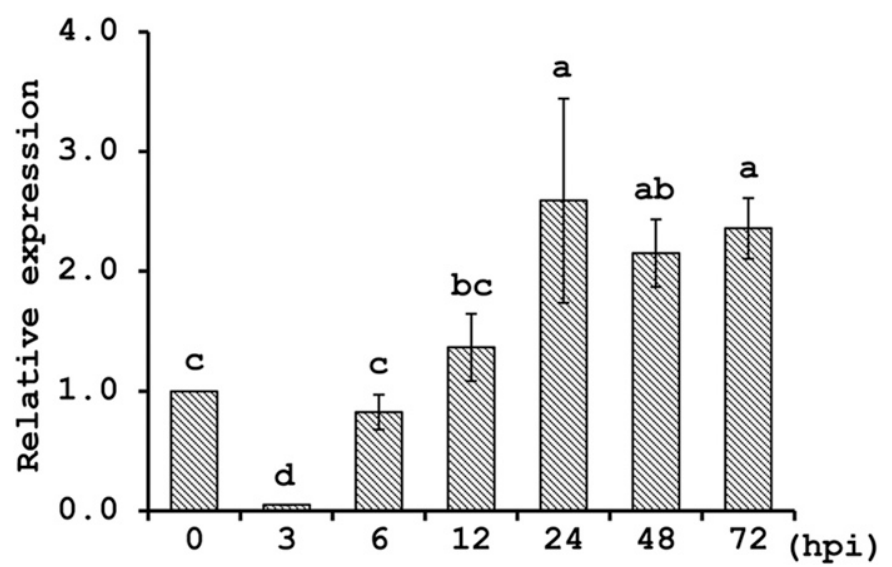

Fig. 1. Expression level of lncRNA39026 during tomato resistance to Phytophthora infestans. Expression level of lncRNA39026 is examined in tomato leaves by quantitative reverse transcription PCR at $0,3,6,12,24,48$, and $72 \mathrm{~h}$ postinoculation (hpi; $n=3$ ).

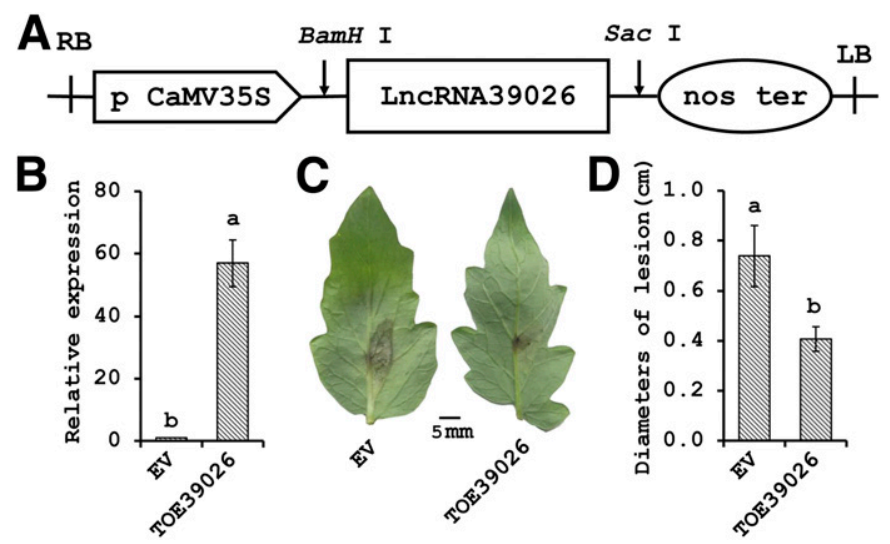

Fig. 2. Overexpression of lncRNA39026 increases tomato resistance to Phytophthora infestans. A, Schematic diagram of pBI121-lncRNA39026 plasmid constructs. B, Expression level of lncRNA39026 in tomato leaves transiently overexpressing lncRNA39026 $(n=3)$. C, Disease symptoms of tomato leaves transiently overexpressing lncRNA39026 at 5 days after inoculation with $P$. infestans. The inoculation position is the back of the tomato leaves. Scale bars = $5 \mathrm{~mm}$. D, Diameter of the lesion of tomato leaves transiently overexpressing lncRNA39026 at 5 days after inoculation with P. infestans $(n=8)$. 
(Cui et al. 2019a) (Fig. 4E). The SlAGO1 accumulation was also examined in tomato leaves infected by $P$. infestans via RT-qPCR. Against the expression level of miR168a, the trend of the expression of SlAGO1 first decreased and then increased, reaching the lowest value at 3 hpi (Fig. 4F). In the tomato leaves in which lncRNA39026 is overexpressed, the expression level of SIAGO1 was upregulated 45-fold (Fig. 4G), whereas SlAGO1 transcripts decreased by approximately $60 \%$ in silenced tomato plant (Fig. 4H). These results indicate that lncRNA39026 functioned as the decoy of miR168a to regulate the expression of SIAGO1.
LncRNA39026 induced the expression of PR genes in tomato. It is well known that PR proteins are the markers of pathogeninduced resistance in plants. To explore whether lncRNA39026 induces the expression of SlPRs, four PR genes, SIPRI (accession number AJ011520), SIPR2 ( $\beta$-1,3-glucanase, accession number M80604), SIPR3 (thaumatinlike/osmotin, accession number Z15141), and SIPR5 (chitinase, accession number AY093595) were selected to examine their transcript levels in tomato plants with overexpressed and silenced lncRNA39026. As shown in Figure 5A, compared with EV plants, the expression levels of
A

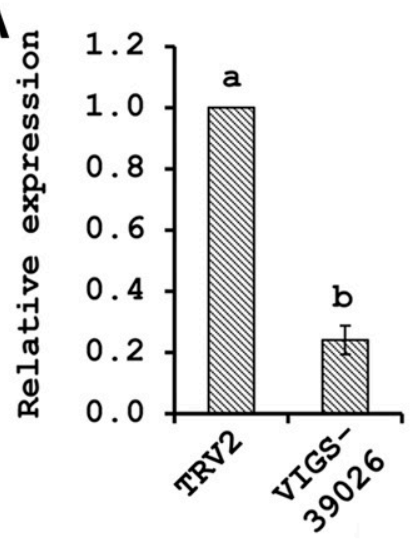

B

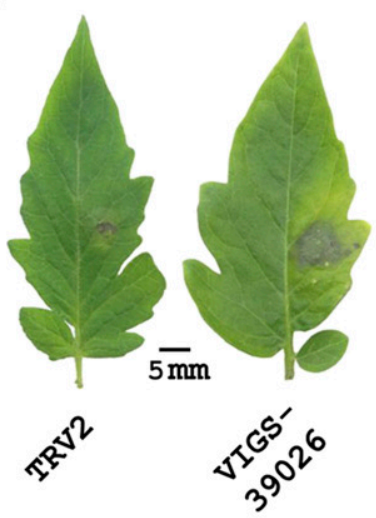

C

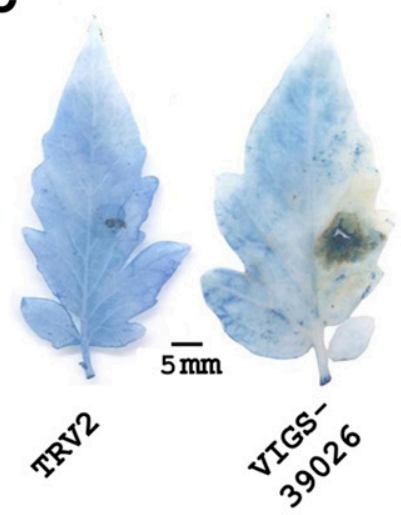

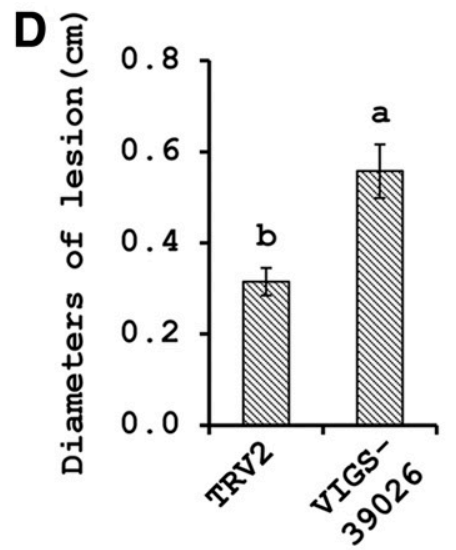

E

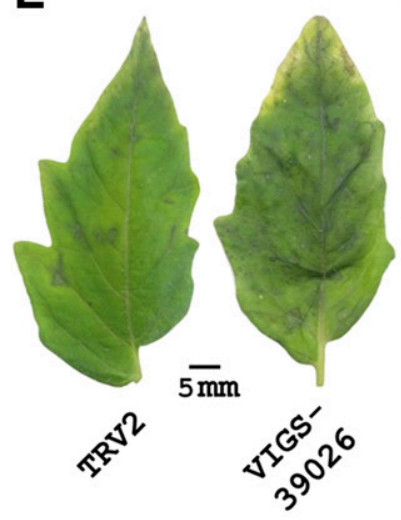

$\mathbf{J}$

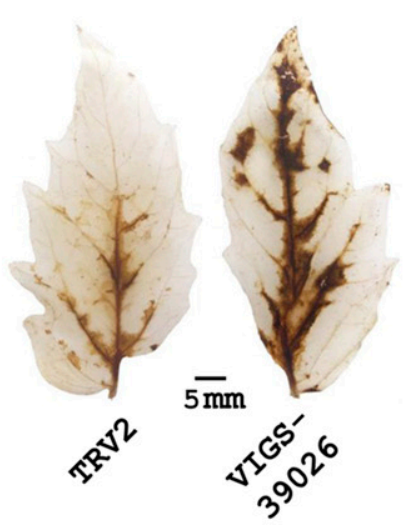

$F$

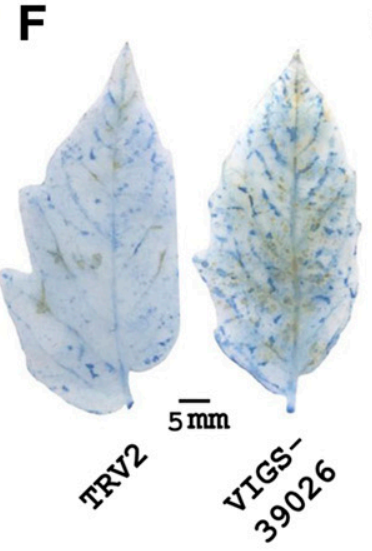

K
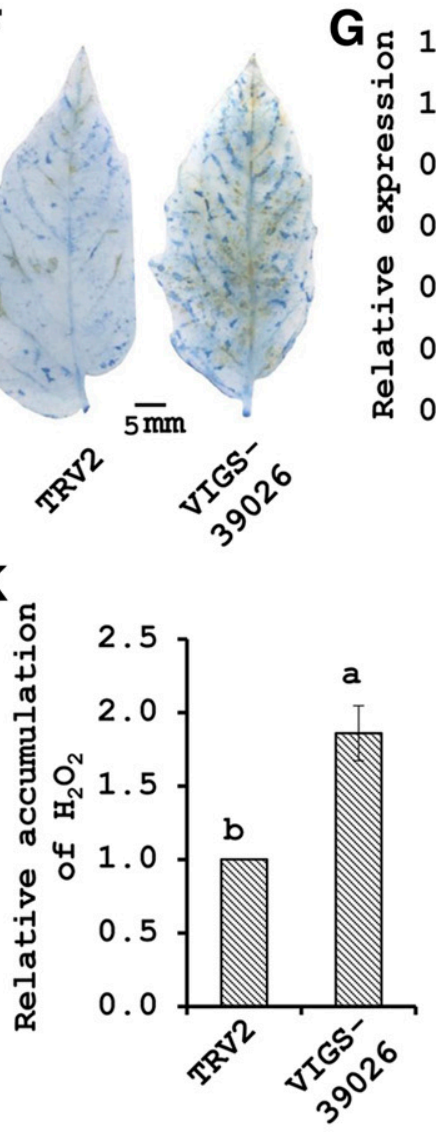

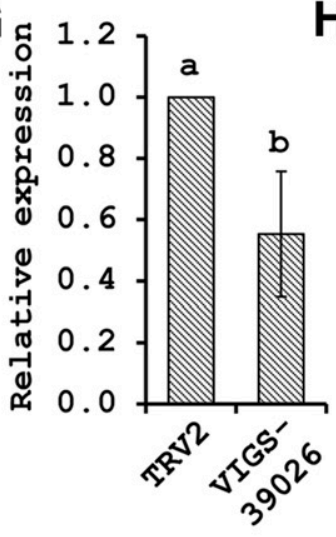

$\mathbf{L}$

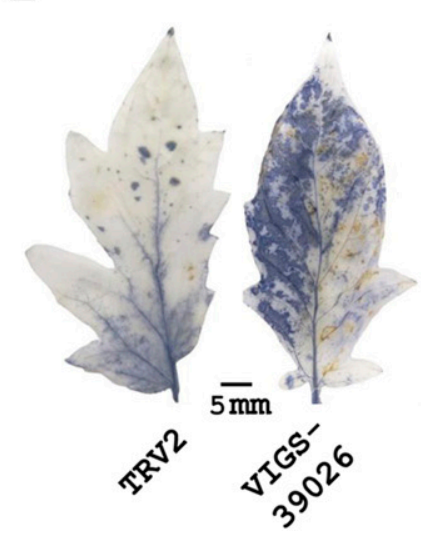

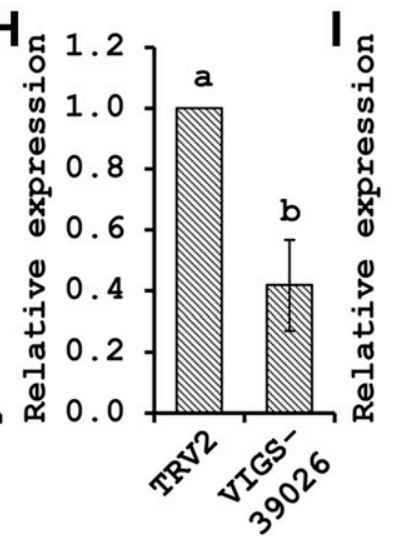

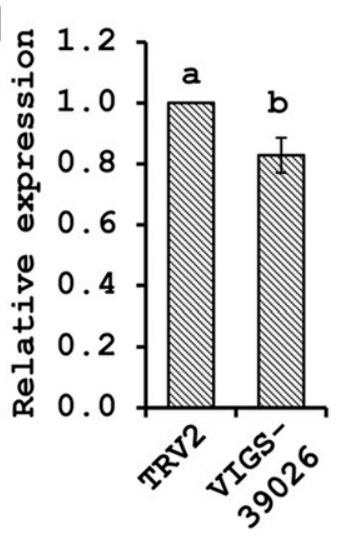

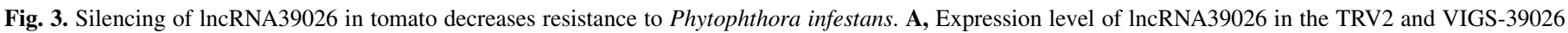

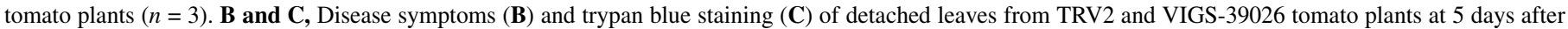

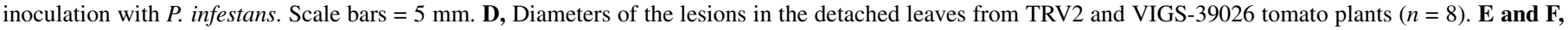

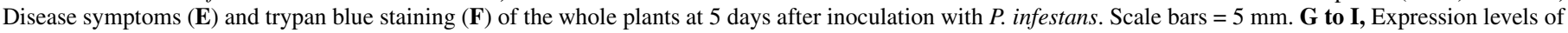

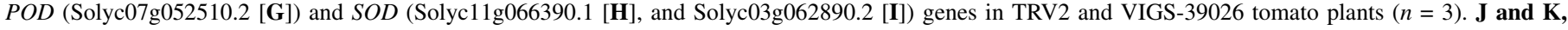

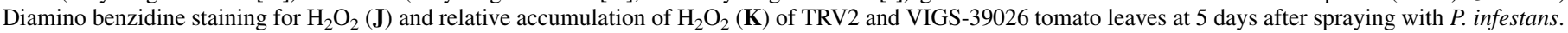

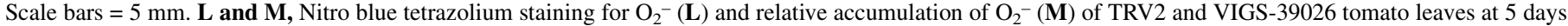
after spraying with $P$. infestans. Scale bars $=5 \mathrm{~mm}$. 
SlPR1, SlPR2, SlPR3, and SlPR5 were upregulated in TOE39026 plants at 3 days after agroinfiltration, approximately 3.2-fold, 1.7fold, 1.6-fold, and 6.9-fold, respectively. After 21 days of VIGS treatment, SlPR1, SlPR2, SlPR3, and SlPR5 in VIGS-39026 tomato plants were downregulated by approximately $32,23,19$, and $68 \%$, respectively (Fig. 5B). These results showed that lncRNA39026 played a regulator role in the expression of PR genes to modulate tomato resistance.

\section{DISCUSSION}

It has been demonstrated that lncRNAs participate in host defense response mechanisms by emerging as new regulators in plants. In our previous studies, many lncRNAs involved in tomato resistance to $P$. infestans have been identified. For example, overexpression of lncRNA16397, lncRNA33732, and lncRNA23468 increases tomato resistance to $P$. infestans, and silencing of lncRNA42705, lncRNA08711, and lncRNA23468 decreases tomato resistance (Cui et al. 2017, 2019a, b; Jiang et al. 2019). As shown in Figure 1, lncRNA39026 was also responsive to $P$. infestans infection. The tomato plants in which IncRNA39026 was overexpressed displayed fewer disease symptoms than tomato plants in which empty vector was overexpressed after infection with $P$. infestans, and silencing of lncRNA39026 decreased tomato resistance, as shown by increased lesion diameters and ROS content (Figs. 2 and 3). These results suggest that lncRNA39026 may increase tomato resistance.

miRNAs also participate in plant defense response. Previous studies have shown that miR168 participates in host defense during pathogen infection. The expression of miR168 is inhibited in Brassica napus infected by Verticillium longisporum (Shen et al. 2014). Also, miR168 is induced in maize by sugarcane mosaic virus infection (Xia et al. 2018). Moreover, miR168a is upregulated when Malus hupehensis is infected by Botryosphaeria dothidea, and its overexpression activates the basal defense and increases resistance to $B$. dothidea (Yu et al. 2017). Upon $P$. infestans infection, the expression level of miR168a also changed, reaching its peak at $3 \mathrm{hpi}$ and its lowest value at $24 \mathrm{hpi}$ (Fig. 4B). MiRNAs function through their target genes. A previous study has demonstrated that miR168 can target the $A G O 1$ gene to participate in plant resistance. A fungal elicitor induces the accumulation of miR168 in Arabidopsis, leading to a consistent decrease in the accumulation of $A G O 1$ transcripts (Baldrich et al., 2014). Moreover, Várallyay et al. (2010)
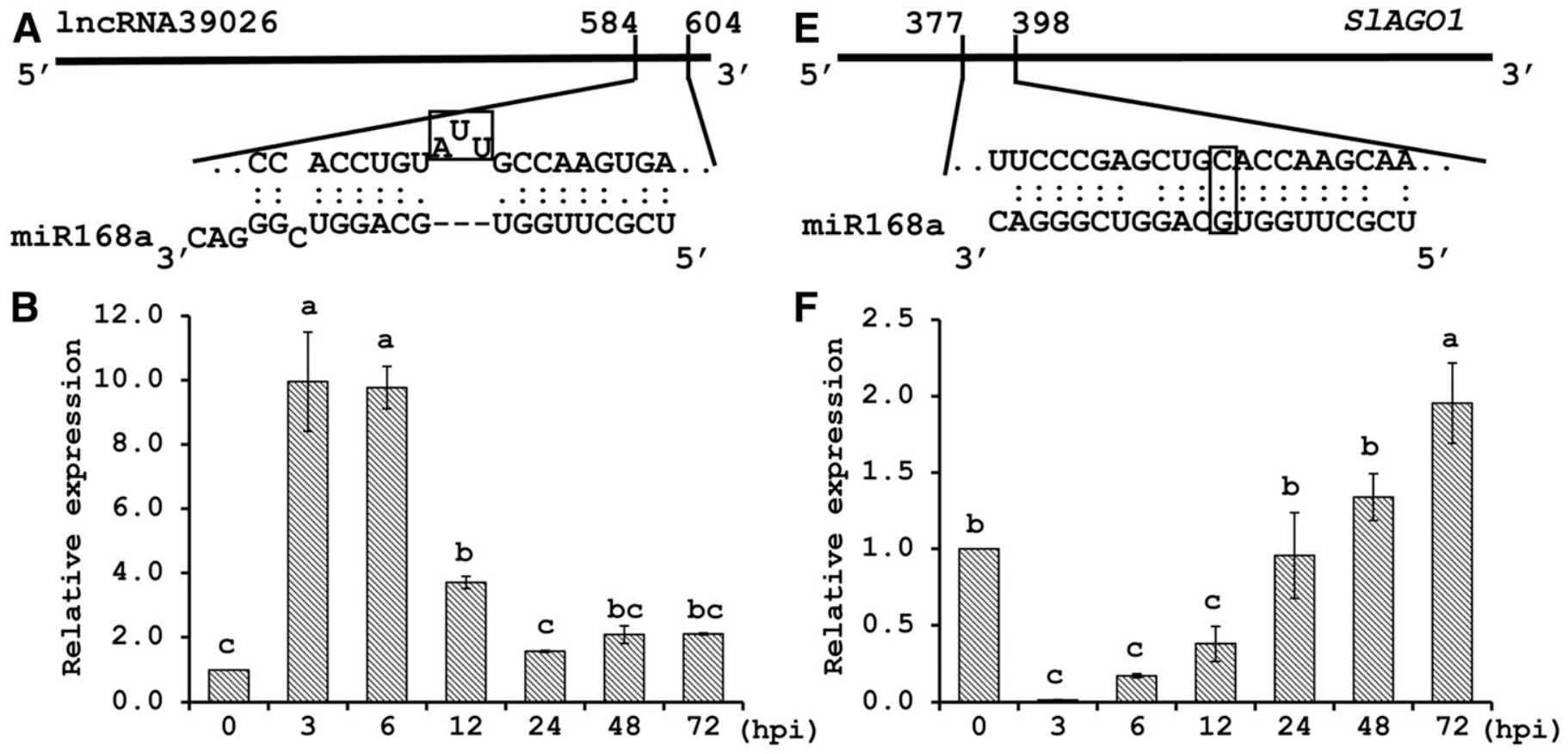

C

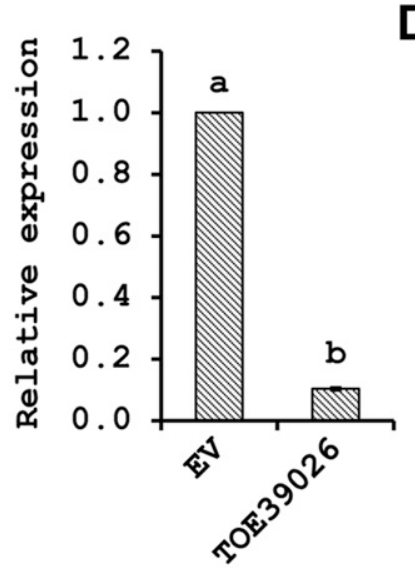

D

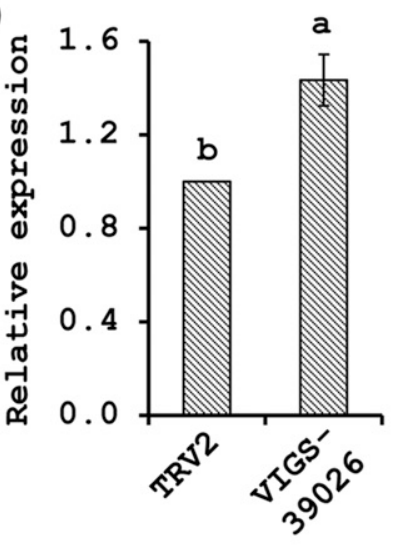

G

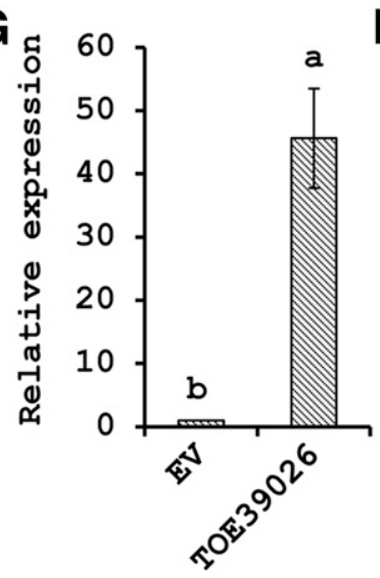

$\mathrm{H}$

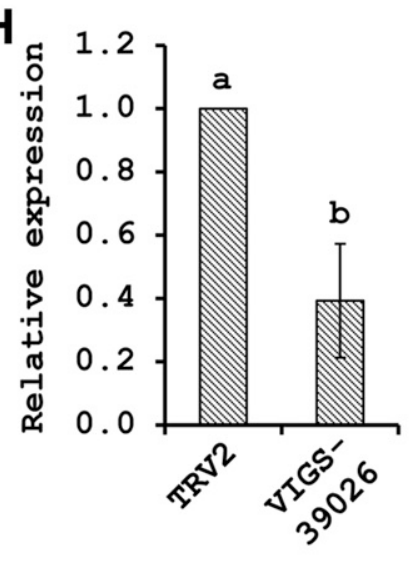

Fig. 4. LncRNA39026 decoys miR168a in tomato. A, Base-pairing interaction between lncRNA39026 and miR168a. Black box indicates bulge. B, Expression patterns of miR168a in tomato leaves at $0,3,6,12,24,48$, and $72 \mathrm{~h}$ postinoculation (hpi). C and D, Expression levels of miR168a in tomato leaves transiently overexpressing (C) and silencing (D) lncRNA39026. E, The miR168a cleavage site of target gene SlAGO1. Black box indicates cleavage sites. F, Expression patterns of SlAGO1 in tomato leaves at 0, 3, 6, 12, 24, 48, and $72 \mathrm{hpi}$. G and $\mathbf{H}$, Expression levels of SlAGO1 in tomato leaves transiently overexpressing (G) and silencing (H) lncRNA39026 $(n=3)$. 
found that an RNA-silencing suppressor induces the expression of miR168 to repress its target accumulation after virus infection. Botrytis cinerea siRNA is transferred into Arabidopsis to silence host AGO1 to reduce plant susceptibility (Weiberg et al. 2013). Our previous study has shown that miR168a cleaves SlAGO1 mRNA in tomato and inhibits its expression to response to $P$. infestans infection (Cui et al. 2019a). In this study, SlAGO1 was also involved in tomato defense response. With $P$. infestans infection, the expression level of SlAGOl underwent changes, which displayed a negative correlation with the expression level of miR168a (Fig. 4F).

Recently, several factors affecting miRNA accumulation have been found. For example, lncRNAs have emerged as a new regulator to affect miRNA expression through the eTM sites for miRNAs in lncRNA sequences (Yang et al. 2019b). Two lncRNAs, slylnc0195 and slylnc1077, contained the eTMs of miR166 and miR399 in tomato and affect tomato resistance to TYLCV by suppressing expression of two miRNAs (Wang et al. 2015). Moreover, three other lncRNAs, IncRNA23468, lncRNA13262, and lncRNA01308, contain the eTM of miR482b. The tomato plants in which lncRNA23468 is overexpressed display a decrease in the expression level of miR $482 \mathrm{~b}$ and an increase in resistance to $P$. infestans, whereas silencing of lncRNA23468 in tomato increases the expression level of miR482b and plant susceptibility (Jiang et al. 2019). Similarly, in this study it was predicted that lncRNA39026 served as an eTM for miR168a. In tomato plants infected with $P$. infestans, the accumulation of lncRNA39026 displayed a negative correlation with the accumulation of miR168a and a positive correlation with the accumulation of SlAGO1 gene (Figs. 1 and 4B and F). Tomato plants in which lncRNA39026 was overexpressed displayed a decreased level of miR168a, an increased level of SlAGO1, and greater resistance to P. infestans (Figs. 2 and 4C and G), whereas, after lncRNA39026 silencing, the accumulation of miR168a and SlAGO1 was decreased and increased, and resistance was impaired (Figs. 3 and 4D and H).

Besides suppressing the expression of miRNAs, lncRNAs also affect the expression of genes. For example, Cui et al. (2017) found that lncRNA16397 can promote glutaredoxin gene expression to alter the tomato resistance to $P$. infestans. Another lncRNA, lncRNA33732 also increases the tomato resistance by inducing the expression of $R B O H$ (Cui et al. 2019b). Moreover, lncRNAs also promote PR genes expression to participate in the plant defense response. A. thaliana lncRNA ELENA1 is designated to increase plant resistance toward $P$. syringe pv. tomato DC3000 by inducing PRl expression (Seo et al. 2017). In addition, in rice infected by Xanthomonas oryzae pv. oryzae, lncRNA ALEX1 has been found to play a regulatory role in plant defense pathways, and its overexpression activates jasmonic acid pathway-related genes including
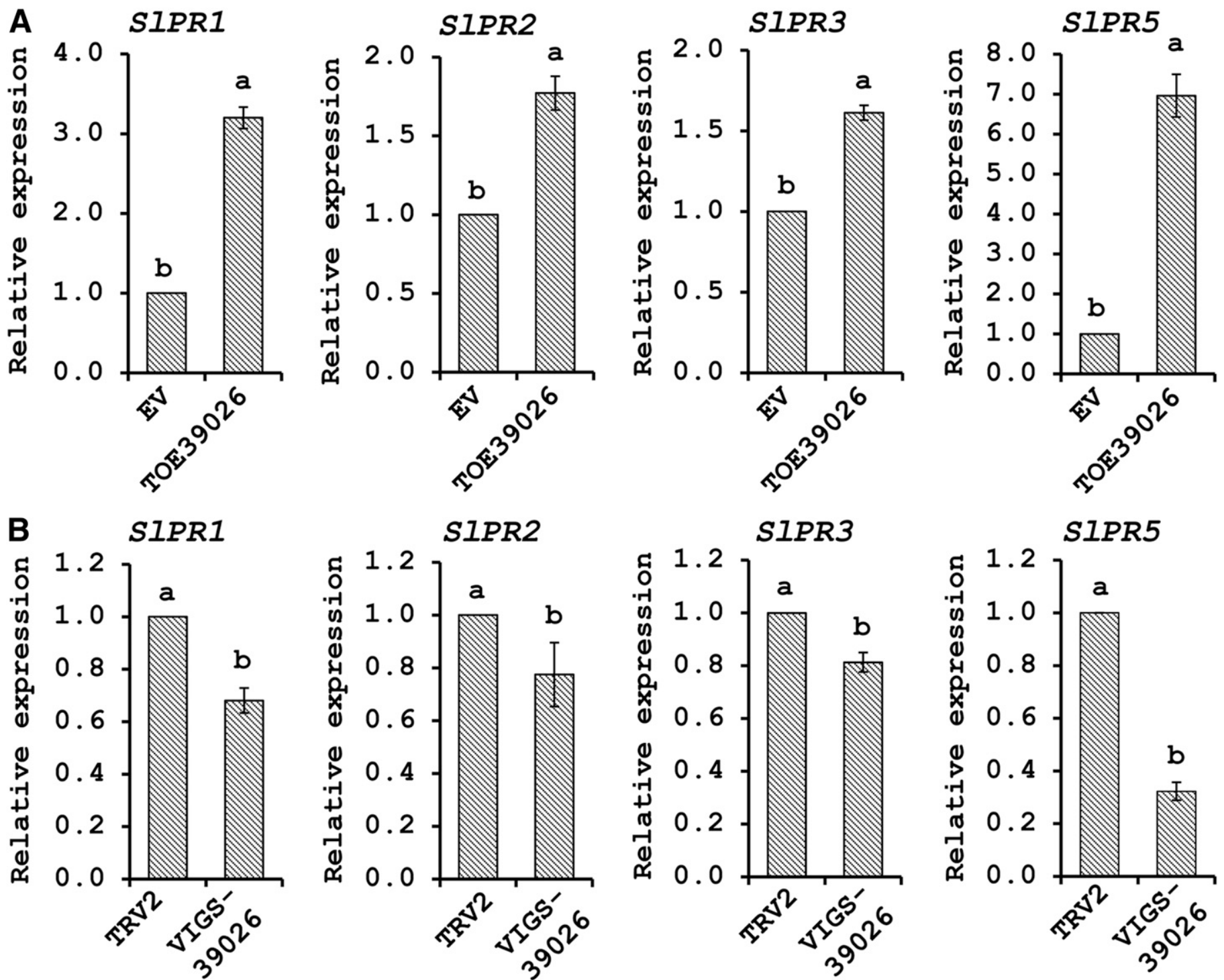

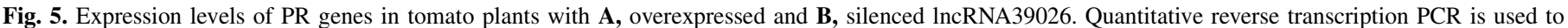
analyze the expression levels of SIPR1, SIPR2, SIPR3, and SIPR5 genes $(n=3)$. 
PR genes to increase rice resistance (Yu et al. 2019). In this study, lncRNA39026 also induced the expression of PR genes. Tomato plants in which lncRNA39026 was overexpressed displayed increased accumulation of SIPR1, SIPR2, SIPR3, and SIPR5 genes (Fig. 5A), whereas silencing of lncRNA39026 inhibited their expression (Fig. 5B). PR proteins play an important role in plant resistance to pathogens. Plants have evolved the ability to systemically defend against pathogens, including systemic acquired resistance (SAR) and induced systemic resistance (ISR) (Grant and Lamb 2006). A set of PR proteins were identified, such as $P R 1, P R 2$, and $P R 5$, as the basic characteristic of SAR signaling pathway (Li et al. 2018) and $P R 3$ as the marker of ISR signaling pathway (Yang et al. 2018). As regards molecular evidence for SAR in tobacco, marker genes such as $P R 1$, $P R 2$, and $P R 5$ are induced by validamycin to increase plant resistance (Ishikawa et al. 2005). Apple PR2 and PR5 have been found to be involved in plant resistance to E. amylovora (Bonasera et al. 2006). Tang et al. (2017) identified an ISR marker gene, $N t P R-Q$, a member of the $P R 3$ family, and its overexpression in tobacco increases resistance to Ralstonia solanacearum. In this study, lncRNA39026 affected the accumulation of PR1, PR2, PR3, and $P R 5$ transcripts (Fig. 5), suggesting lncRNA39026 may be involved in SAR and ISR signaling pathways to regulate tomato resistance.

Based on our results, we constructed a model explaining how lncRNA39026 confers tomato resistance toward P. infestans (Fig. 6). Following from our results for lncRNA39026 overexpressing and silencing tomato plants, we propose that lncRNA39026 positively regulates the tomato defense response by acting as an eTM for miR168a to regulate SlAGO1. Also, lncRNA36026 is involved in SAR and ISR by affecting the accumulation of PR genes. Our results demonstrate that the lncRNA39026-mediated interaction influences tomato resistance to $P$. infestans and perfects regulation mechanism of lncRNAs in plant defense responses to pathogen infection.

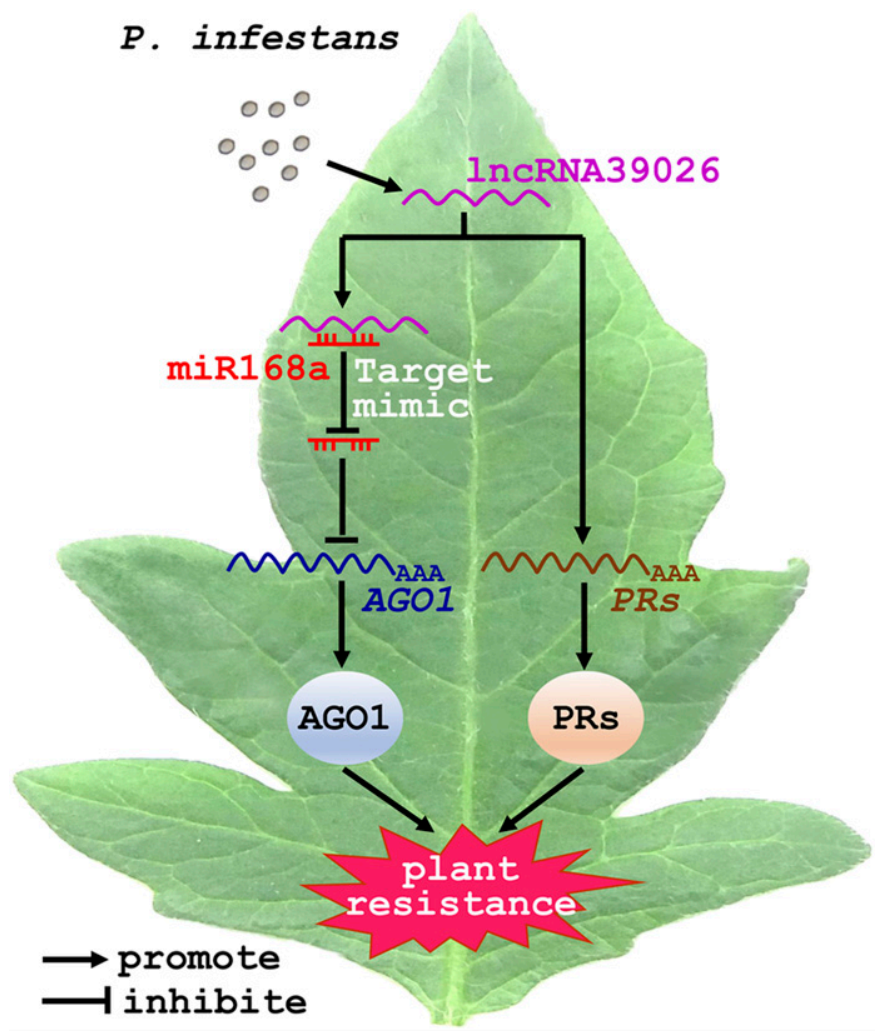

Fig. 6. Model of the lncRNA39026-mediated regulatory mechanism during tomato resistance to Phytophthora infestans.

\section{LITERATURE CITED}

Baldrich, P., Kakar, K., Siré, C., Moreno, A. B., Berger, A., García-Chapa, M., López-Moya, J. J., Riechmann, J. L., and San Segundo, B. 2014. Small RNA profiling reveals regulation of Arabidopsis miR168 and heterochromatic siRNA415 in response to fungal elicitors. BMC Genomics 15:1083.

Baysal-Gurel, F., Li, R., Ling, K. S., and Miller, S. A. 2015. First report of tomato chlorotic spot virus infecting tomatoes in Ohio. Plant Dis. 99:163.

Bonasera, J. M., Kim, J. F., and Beer, S. V. 2006. PR genes of apple: Identification and expression in response to elicitors and inoculation with Erwinia amylovora. BMC Plant Biol. 6:23.

Cui, J., Jiang, N., Hou, X., Wu, S., Zhang, Q., Meng, J., and Luan, Y. 2019a. Genome-wide identification of lncRNAs and analysis of ceRNA networks during tomato resistance to Phytophthora infestans. Phytopathology doi: 10.1094/PHYTO-04-19-0137-R.

Cui, J., Jiang, N., Meng, J., Yang, G., Liu, W., Zhou, X., Ma, N., Hou, X., and Luan, Y. 2019b. LncRNA33732-respiratory burst oxidase module associated with WRKY1 in tomato-Phytophthora infestans interactions. Plant J. 97:933-946

Cui, J., Luan, Y., Jiang, N., Bao, H., and Meng, J. 2017. Comparative transcriptome analysis between resistant and susceptible tomato allows the identification of lncRNA16397 conferring resistance to Phytophthora infestans by co-expressing glutaredoxin. Plant J. 89:577-589.

Cui, J., Xu, P., Meng, J., Li, J., Jiang, N., and Luan, Y. 2018. Transcriptome signatures of tomato leaf induced by Phytophthora infestans and functional identification of transcription factor SpWRKY3. Theor. Appl. Genet. 131: 787-800.

Dai, L., Wang, D., Xie, X., Zhang, C., Wang, X., Xu, Y., Wang, Y., and Zhang, J. 2016. The novel gene VpPR4-1 from Vitis pseudoreticulata increases powdery mildew resistance in transgenic Vitis vinifera L. Front. Plant Sci. 7: 695.

Deng, F., Zhang, X., Wang, W., Yuan, R., and Shen, F. 2018. Identification of Gossypium hirsutum long non-coding RNAs (lncRNAs) under salt stress. BMC Plant Biol. 18:23.

Du, Q., Wang, K., Zou, C., Xu, C., and Li, W. 2018. The PILNCR1-miR399 regulatory module is important for low phosphate tolerance in maize. Plant Physiol. 177:1743-1753.

Feng, K., Yu, J., Cheng, Y., Ruan, M., Wang, R., Ye, Q., Zhou, G., Li, Z., Yao, Z., Yang, Y., Zheng, Q., and Wan, H. 2016. The SOD gene family in tomato: identification, phylogenetic relationships, and expression patterns. Front. Plant Sci. 7:1279.

Franco-Zorrilla, J. M., Valli, A., Todesco, M., Mateos, I., Puga, M. I., Rubio-Somoza, I., Leyva, A., Weigel, D., García, J. A., and Paz-Ares, J. 2007. Target mimicry provides a new mechanism for regulation of microRNA activity. Nat. Genet. 39:1033-1037.

Gao, L., Gonda, I., Sun, H., Ma, Q., Bao, K., Tieman, D. M., Burzynski-Chang, E. A., Fish, T. L., Stromberg, K. A., Sacks, G. L., Thannhauser, T. W., Foolad, M. R., Diez, M. J., Blanca, J., Canizares, J., Xu, Y., van der Knaap, E., Huang, S., Klee, H. J., Giovannoni, J. J., and Fei, Z. 2019. The tomato pan-genome uncovers new genes and a rare allele regulating fruit flavor. Nat. Genet. 51:1044-1051.

Grant, M., and Lamb, C. 2006. Systemic immunity. Curr. Opin. Plant Biol. 9: 414-420.

Hassani, M., Salami, S. A., Nasiri, J., Abdollahi, H., and Ghahremani, Z. 2016. Phylogenetic analysis of $P R$ genes in some pome fruit species with the emphasis on transcriptional analysis and ROS response under Erwinia amylovora inoculation in apple. Genetica 144:9-22.

Hong, Y., Cui, J., Liu, Z., and Luan, Y. 2018. SpWRKY6 acts as a positive regulator during tomato resistance to Phytophthora infestans infection. Biochem. Biophys. Res. Commun. 506:787-792.

Ishikawa, R., Shirouzu, K., Nakashita, H., Lee, H., Motoyama, T., Yamaguchi, I., Teraoka, T., and Arie, T. 2005. Foliar spray of validamycin a or validoxylamine a controls tomato fusarium wilt. Phytopathology 95:1209-1216.

Jiang, N., Cui, J., Meng, J., and Luan, Y. 2018a. A tomato nucleotide binding sites-leucine-rich repeat gene is positively involved in plant resistance to Phytophthora infestans. Phytopathology 108:980-987.

Jiang, N., Cui, J., Shi, Y., Yang, G., Zhou, X., Hou, X., Meng, J., and Luan, Y. 2019. Tomato lncRNA23468 functions as a competing endogenous RNA to modulate NBS-LRR genes by decoying miR482b in the tomato-Phytophthora infestans interaction. Hortic. Res. 6:28.

Jiang, N., Meng, J., Cui, J., Sun, G., and Luan, Y. 2018b. Function identification of miR482b, a negative regulator during tomato resistance to Phytophthora infestans. Hortic. Res. 5:9.

Lavrova, V. V., Zinovieva, S. V., Udalova, Z. V., and Matveeva, E. M. 2017. Expression of $P R$ genes in tomato tissues infected by nematode Meloidogyne incognita (Kofoid et White, 1919) Chitwood, 1949. Dokl. Biochem. Biophys. 476:306-309.

Li, J., Luan, Y., and Liu, Z. 2015. SpWRKY1 mediates resistance to Phytophthora infestans and tolerance to salt and drought stress by modulating 
reactive oxygen species homeostasis and expression of defense-related genes in tomato. Plant Cell Tissue Organ Cult. 123:67-81.

Li, Y., Lu, Y., Shi, Y., Wu, L., Xu, Y., Huang, F., Guo, X., Zhang, Y., Fan, J., Zhao, J., Zhang, H., Xu, P., Zhou, J., Wu, X., Wang, P., and Wang, W. 2014. Multiple rice microRNAs are involved in immunity against the blast fungus Magnaporthe oryzae. Plant Physiol. 164:1077-1092.

Li, Y., Zhang, W., Dong, H., Liu, Z., Ma, J., and Zhang, X. 2018. Salicylic acid in Populus tomentosa is a remote signalling molecule induced by Botryosphaeria dothidea infection. Sci. Rep. 8:14059.

Luan, Y., Cui, J., Zhai, J., Li, J., Han, L., and Meng, J. 2015. High-throughput sequencing reveals differential expression of miRNAs in tomato inoculated with Phytophthora infestans. Planta 241:1405-1416.

Pertermann, R., Tamilarasan, S., Gursinsky, T., Gambino, G., Schuck, J., Weinholdt, C., Lilie, H., Grosse, I., Golbik, R. P., Pantaleo, V., and Behrens, S. E. 2018. A viral suppressor modulates the plant immune response early in infection by regulating microRNA activity. MBio 9:e00419-18.

Ren, Z., You, Z., Munir, S., Zhang, Y., Li, H., Zhang, J., Wang, J., Wang, T., Zheng, W., and Ye, Z. 2019. Development of a highly specific codominant marker for genotyping the $P h-3$ (tomato late blight resistance) locus by comparing cultivated and wild ancestor species. Mol. Breed. 39:45.

Seo, J. S., Sun, H. X., Park, B. S., Huang, C., Yeh, S. D., Jung, C., and Chua, N. H. 2017. ELF18-INDUCED LONG-NONCODING RNA associates with mediator to enhance expression of innate immune response genes in Arabidopsis. Plant Cell 29:1024-1038.

Shen, D., Suhrkamp, I., Wang, Y., Liu, S., Menkhaus, J., Verreet, J., Fan, L., and Cai, D. 2014. Identification and characterization of microRNAs in oilseed rape (Brassica napus) responsive to infection with the pathogenic fungus Verticillium longisporum using Brassica AA (Brassica rapa) and CC (Brassica oleracea) as reference genomes. New Phytol. 204:577-594.

Tang, Y., Liu, Q., Liu, Y., Zhang, L., and Ding, W. 2017. Overexpression of $N t P R-Q$ up-regulates multiple defense-related genes in Nicotiana tabacum and enhances plant resistance to Ralstonia solanacearum. Front. Plant Sci. $8: 1963$.

Várallyay, E., and Havelda, Z. 2013. Unrelated viral suppressors of RNA silencing mediate the control of ARGONAUTE1 level. Mol. Plant Pathol. 14:567-575

Várallyay, E., Válóczi, A., Agyi, A., Burgyán, J., and Havelda, Z. 2010. Plant virus-mediated induction of miR168 is associated with repression of ARGONAUTE1 accumulation. EMBO J. 29:3507-3519.

Vaucheret, H. 2009. AGO1 homeostasis involves differential production of 21-nt and 22-nt miR168 species by MIR168a and MIR168b. PLoS One 4: e6442.

Voinnet, O. 2009. Origin, biogenesis, and activity of plant microRNAs. Cell 136:669-687.

Wang, A., Hu, J., Gao, C., Chen, G., Wang, B., Lin, C., Song, L., Ding, Y., and Zhou, G. 2019. Genome-wide analysis of long non-coding RNAs unveils the regulatory roles in the heat tolerance of Chinese cabbage (Brassica rapa ssp.chinensis). Sci. Rep. 9:5002.

Wang, J., Yang, Y., Jin, L., Ling, X., Liu, T., Chen, T., Ji, Y., Yu, W., and Zhang, B. 2018. Re-analysis of long non-coding RNAs and prediction of circRNAs reveal their novel roles in susceptible tomato following TYLCV infection. BMC Plant Biol. 18:104.

Wang, J., Yu, W., Yang, Y., Li, X., Chen, T., Liu, T., Ma, N., Yang, X., Liu, R., and Zhang, B. 2015. Genome-wide analysis of tomato long non-coding RNAs and identification as endogenous target mimic for microRNA in response to TYLCV infection [published correction appears in Sci Rep. 6: 32828]. Sci. Rep. 5:16946.
Wang, M., Wu, H. J., Fang, J., Chu, C., and Wang, X. 2017. A long noncoding RNA involved in rice reproductive development by negatively regulating osa-miR160. Sci. Bull. (Beijing) 62:470-475.

Wang, X., Ai, G., Zhang, C., Cui, L., Wang, J., Li, H., Zhang, J., and Ye, Z. 2016. Expression and diversification analysis reveals transposable elements play important roles in the origin of Lycopersicon-specific lncRNAs in tomato. New Phytol. 209:1442-1455.

Weiberg, A., Wang, M., Lin, F., Zhao, H., Zhang, Z., Kaloshian, I., Huang, H., and Jin, H. 2013. Fungal small RNAs suppress plant immunity by hijacking host RNA interference pathways. Science 342:118-123.

Wierzbicki, A. T. 2012. The role of long non-coding RNA in transcriptional gene silencing. Curr. Opin. Plant Biol. 15:517-522.

Wu, H., Wang, Z., Wang, M., and Wang, X. 2013. Widespread long noncoding RNAs as endogenous target mimics for microRNAs in plants. Plant Physiol. 161:1875-1884

Xia, Z., Zhao, Z., Li, M., Chen, L., Jiao, Z., Wu, Y., Zhou, T., Yu, W., and Fan, Z. 2018. Identification of miRNAs and their targets in maize in response to Sugarcane mosaic virus infection. Plant Physiol. Biochem. 125:143-152.

Yang, Y., Chen, T., Ling, X., and Ma, Z. 2018. Gbvdr6, a gene encoding a receptor-like protein of cotton (Gossypium barbadense), confers resistance to Verticillium wilt in Arabidopsis and upland cotton. Front. Plant Sci. 8:2272.

Yang, Y., Liu, T., Shen, D., Wang, J., Ling, X., Hu, Z., Chen, T., Hu, J., Huang, J., Yu, W., Dou, D., Wang, M., and Zhang, B. 2019a. Tomato yellow leaf curl virus intergenic siRNAs target a host long noncoding RNA to modulate disease symptoms. PLoS Pathog 15:e1007534.

Yang, Z., Yang, C., Wang, Z., Yang, Z., Chen, D., and Wu, Y. 2019b. LncRNA expression profile and ceRNA analysis in tomato during flowering. PLoS One 14:e0210650.

Yu, X., Hou, Y., Chen, W., Wang, S., Wang, P., and Qu, S. 2017. Malus hupehensis miR168 targets to ARGONAUTE1 and contributes to the resistance against Botryosphaeria dothidea infection by altering defense responses. Plant Cell Physiol. 58:1541-1557.

Yu, Y., Zhou, Y., Feng, Y., He, H., Lian, J., Yang, Y., Lei, M., Zhang, Y., and Chen, Y. 2019. Transcriptional landscape of pathogen-responsive lncRNAs in rice unveils the role of ALEX1 in jasmonate pathway and disease resistance. Plant Biotechnol. J. pbi.13234.

Zhang, C., Liu, L., Zheng, Z., Sun, Y., Zhou, L., Yang, Y., Cheng, F., Zhang, Z., Wang, X., Huang, S., Xie, B., Du, Y., Bai, Y., and Li, J. 2013a. Fine mapping of the $P h-3$ gene conferring resistance to late blight (Phytophthora infestans) in tomato. Theor. Appl. Genet. 126:2643-2653.

Zhang, H., Chen, X., Wang, C., Xu, Z., Wang, Y., Liu, X., Kang, Z., and Ji, W. 2013b. Long non-coding genes implicated in response to stripe rust pathogen stress in wheat (Triticum aestivum L.). Mol. Biol. Rep. 40:6245-6253.

Zhang, H., Hu, W., Hao, J., Lv, S., Wang, C., Tong, W., Wang, Y., Wang, Y., Liu, X., and Ji, W. 2016. Genome-wide identification and functional prediction of novel and fungi-responsive lincRNAs in Triticum aestivum. BMC Genomics 17:238.

Zhang, L., Wang, M., Li, N., Wang, H., Qiu, P., Pei, L., Xu, Z., Wang, T., Gao, E., Liu, J., Liu, S., Hu, Q., Miao, Y., Lindsey, K., Tu, L., Zhu, L., and Zhang, X. 2018. Long noncoding RNAs involve in resistance to Verticillium dahliae, a fungal disease in cotton. Plant Biotechnol. J. 16:1172-1185.

Zheng, Y., Wang, Y., Ding, B., and Fei, Z. 2017. Comprehensive transcriptome analyses reveal that Potato Spindle Tuber Viroid triggers genome-wide changes in alternative splicing, inducible trans-acting activity of phased secondary small interfering RNAs, and immune responses. J. Virol. 91: e00247-17.

Zhu, Q., Stephen, S., Taylor, J., Helliwell, C. A., and Wang, M. 2014. Long noncoding RNAs responsive to Fusarium oxysporum infection in Arabidopsis thaliana. New Phytol. 201:574-584. 